\title{
Assessment of the hand in cerebral palsy
}

\author{
Praveen Bhardwaj, S. Raja Sabapathy \\ Department of Plastic Surgery, Hand Surgery, Reconstructive Microsurgery and Burns Ganga Hospital, 313, Mettupalayam \\ Road, Coimbatore, India
}

Address for correspondence: Dr. S. Raja Sabapathy, Department of Plastic Surgery, Hand Surgery, Reconstructive Microsurgery and Burns, Ganga Hospital, 313 Mettupalayam Road, Coimbatore- 641 043, India. E-mail: rajahand@vsnl.com

\section{ABSTRACT}

Cerebral palsy is the musculoskeletal manifestation of a nonprogressive central nervous system lesion that usually occurs due to a perinatal insult to the brain. Though the cerebral insult is static the musculoskeletal pathology is progressive. Some patients with cerebral palsy whose hands are affected can be made better by surgery. The surgical procedures as such are not very technically demanding but the assessment, decision-making, and selecting the procedures for the given patient make this field challenging. When done well, the results are rewarding not only in terms of improvement in hand function but also in appearance and personal hygiene, which leads to better self-image and permits better acceptance in the society. This article focuses on the clinical examination, patient selection, and decision-making while managing these patients.

\section{KEY WORDS}

Assessment, cerebral palsy, decision-making, examination, spastic hand

\section{ASSESSMENT OF THE HAND IN CEREBRAL PALSY}

erebral palsy is the musculoskeletal manifestation of a nonprogressive central nervous system lesion that usually occurs due to a perinatal insult to the brain. Though the cerebral insult is static the musculoskeletal pathology is progressive. Spasticity leads to shortening of musculoskeletal units, which in turn causes fixed contractures and eventually leads to torsional abnormalities of long bones, joint instability, deformity, and degenerative arthritis. ${ }^{[1]}$ The clinical manifestation may vary widely ranging from an intelligent child with

\begin{tabular}{|l|l|}
\hline \multicolumn{2}{|c|}{ Access this article online } \\
\hline Quick Response Code: & Website: \\
\hline & www.ijps.org \\
\cline { 2 - 2 } & Dol: \\
\hline
\end{tabular}

Indian Journal of Plastic Surgery May-August 2011 Vol 44 Issue 2 mild spasticity of the hand to a completely wheelchair bound child who is unable to communicate and totally ignores his involved upper limb. But all patients have these common features: ${ }^{[2]}$

- Cerebral palsy is the result of a brain lesion. Therefore, the spinal cord and muscles are structurally and biochemically normal.

- The brain lesion must be fixed and nonprogressive. Thus, all of the progressive neurodegenerative disorders are excluded from the definition.

- The abnormality of the brain results in motor impairment.

The complexity of the spastic hand is due to the fact that the spastic muscles cannot be used as tendon transfers with the same efficiency as done in reconstructive surgery of the hand with flaccid paralysis. ${ }^{[3]}$ The deformity is basically a dynamic deformity and hence the surgeon must have a definite plan before embarking on the procedure. After anesthesia, the deformities disappear 
and the hand may appear normal. The senior author would always say that "In cerebral palsy the consultant cannot operate based on the findings of the registrar. One needs to know the extent of release required, tension adjustment in tendon transfer, stability of the joints the proposed tendon transfers would cross, the spasticity and voluntary control of the muscles selected for the transfer and moreover the child's use of the hand during the bimanual activities." The surgical procedures as such are not very technically demanding but the assessment, decision-making, and selecting a procedure for the given patient make this field challenging. When done well, the results are rewarding not only in terms of improvement in hand function but also in appearance and personal hygiene, which leads to better self-image and permits better acceptance in the society.

The goals of surgical intervention are set depending on the preoperative functional status. When the child has a good voluntary motor control the goals are to improve function and appearance. In cases of severe involvement, surgery is a reasonable option if it facilitates the nursing care by the parents or the care giver.

\section{DIAGNOSING CEREBRAL PALSY}

Delay in milestones is an important indicator of cerebral palsy. So a surgeon should be familiar with the normal infant milestones. On average head control in an infant is attained at 3-4 months, sitting by 6 months, crawling by 9 months, standing by 10-12 months, and walking by $12-18$ months. The diagnosis should be suspected if there is paucity of spontaneous movements during the first few months of life, or if the motion of both upper limbs is not symmetric. Preferential use of one hand is an important signal toward impaired neurologic status of the other limb. Normally the hand dominance starts developing at about 2-3 years and is fully developed by 6 years. Early handedness, particularly apparent lefthandedness in small infants, is often a clue that the neurologic status of the other extremity is abnormal, and that spastic hemiplegia may be present. ${ }^{[2]}$ Infantile reflexes like Moro's reflex, parachute reflex, tonic neck reflex are normal in a neonate but disappear by 3--6 months as the motor cortex matures and overrides them. ${ }^{[2]}$ These reflexes are retained in children with cerebral palsy. Identification of upper limb dysfunction is usually noted by 1 year of age. Normally at 1 year an infant develops a refined pinch with opposition of the thumb to the index finger but a child with cerebral palsy does not reach this milestone, although they may develop a more primitive key pinch (thumb to side of index finger). ${ }^{[4]}$ When the child is older, sensory defects, abnormal intelligence, and poor voluntary control of various muscle groups become obvious. If the child was born full term, if there were no perinatal medical problems, and especially if the child began to develop normally and then regressed, prompt neurologic consultation should be sought. The neurologist will differentiate cerebral palsy from brain and spinal cord tumors and progressive neurodegenerative diseases. ${ }^{[2]}$

\section{OUTCOME OF SURGERY FOR UPPER EXTREMITY IN CEREBRAL PALSY}

Outcome of surgery in the upper limb cerebral palsy is not as good as in lower limbs because children preferentially use the good limb for most activities and it is difficult to train a limb suffering from "learned non-use." Retraining the upper limb is more difficult because it lacks repetitive motor tasks, like walking. Higher incidence of dystonia, weakness, sensory impairment, and poor selective motor control coexist with spasticity in the upper limb. Compared with lower limb, upper limb tasks are more sophisticated and require high level of coordination and control.

\section{CLINICAL ASSESSMENT}

The examination of a child with cerebral palsy requires patience. An examination of the motor, sensory, and intellectual function is done. Multiple visits may be needed. Symptoms may vary with the child's emotional state and fatigue level. Lengthy examinations should be fragmented. Video recording is helpful.

The child and family are asked to describe precisely how the hand is used in activities of daily living, such as dressing, self-care, and eating. Parents are usually able to state the functional and cosmetic disability the child has.

The motor examination of the upper limb should be aimed to address these six points:

- The dominant and deforming spastic muscles, their strength, and voluntary control.

- The status of the weak or nonfunctioning antagonists.

- Potential donors to substitute the weak muscles.

- Global motor control of the upper limb.

Indian Journal of Plastic Surgery May-August 2011 Vol 44 Issue 2 
- Voluntary control of various muscle groups.

- Existing function and functional needs of the hand for that particular patient.

The examination begins with observing the position of the rest of the extremity. The position of the limb should also be assessed in walking and running which represents the actual cosmetic and functional disability.

Resting position provides information on the amount of spasticity. Spasticity of the muscle can be detected by performing the passive opposite movement and feeling for the abnormal contraction of the muscles. Spasticity of finger extrinsic muscles can be tested by tapping briefly on the pulps of the fingers (for flexors), on the nails (for extensors), which produces an exaggerated response of the tested muscles. ${ }^{[5]}$ Spasticity of the intrinsic muscles of the hand is extremely difficult to evaluate because of the associated deformities. Generally a swan-neck deformity with the associated flexion of the metacarpophalangeal (MCP) joint indicates intrinsic spasticity. The spastic muscle is usually active, but their voluntary movements are often impaired by cocontractions, e.g., cocontraction of the wrist flexors with the attempted finger flexion.

An attempt is made to grade the power of the spastic muscle though it is not always accurate. Passive as well as active range of motion of all joints should be measured. It is important to differentiate muscle spasticity from muscle and joint contracture. Muscle spasticity can be overcome by the application of gentle sustained resistance to the spastic force, whereas muscle contracture cannot. Muscle spasticity, with relaxation, allows a full range of motions of the joint.

The nonfunctioning or the weak muscles are identified and the strength of all potential donor muscles for transfer should be measured. In some cases the muscle may be present but made ineffective by the spastic antagonist or by the elongation caused by a severe deformity. Careful palpation of the muscle belly during attempted active motion may confirm that the muscle is not paralyzed but cannot give information of its actual strength. Asking patients to perform activities with both extremities simultaneously can ensure that the patient comprehends instructions.

Global motor control can be evaluated by standard tests, such as head-to-knee test, in which the patient is asked to place his hand on his head and then move it to the contralateral knee. The speed and precision of the movement are recorded.

Volitional use of the hand is the best predictor of functional improvement after a change in position of the hand. Each muscle or a group of muscles are evaluated for voluntary motor control.

\section{Grasp and release}

The child is asked to pick up the blocks of different size and shape. This test evaluates not only the prehensile capacity of the hand but also the contribution of the whole limb to that function. Quantitative measurements can be made if one introduces the time factor.

\section{Bimanual activities}

This gives accurate information of the spastic upper limb's actual functional ability.

\section{GRADING OF HAND FUNCTION}

McConnell and colleagues ${ }^{[6]}$ reviewed 18 classification systems for the upper limb in children with cerebral palsy, and found House classification reliable and clinically useful. House's ${ }^{[7]}$ classification contains nine subgroups and gives detailed functional levels. It makes identification of small functional improvement possible [Table 1]. A simpler but equally useful classification of practical use in clinical practice is the system of Green and Banks, ${ }^{[8]}$ modified by Samilson and Morris, ${ }^{[9]}$ which contains four subgroups:

- Poor: Use of the hand only as a paperweight, poor or absent grasp and release, and poor control

- Fair: Use of the hand as a helping hand but no effectual use of the hand in dressing, moderate grasp and release, and fair control

- Good: Use of the hand as a help in dressing and eating and general activities, effectual grasp and release, excellent control

- Excellent: Good use of the hand in dressing and eating, effectual grasp and release, and good control

\section{CRITERIA FOR PATIENT SELECTION FOR SURGICAL INTERVENTION}

\section{Age for surgery}

The ideal age for the surgery is debatable. Four to six years is recommended because at this age adequate maturation of the nervous system has occurred. ${ }^{[10]}$ At 4 
Table 1: House's ${ }^{[7]}$ functional classification system

\begin{tabular}{lll}
\hline Class & Designation & Activity level \\
\hline 0 & Does not use & Does not use \\
1 & Poor passive assist & Uses as stabilizing weight only \\
2 & Fair passive assist & Can hold onto object placed in hand \\
3 & Good passive assist & Can hold onto object and stabilize it for use by other hand \\
4 & Poor active assist & Can actively grasp object and hold it weakly \\
5 & Fair active assist & Can actively grasp object and stabilize it well \\
6 & Good active assist & Can actively grasp object and then manipulate it against other hand \\
7 & Spontaneous use & Can perform bimanual activities easily and occasionally uses the hand spontaneously \\
8 & Spontaneous use & Uses hand completely independently without reference to the other hand \\
\hline
\end{tabular}

years the deformities are easily detected and the child is usually mature and motivated enough to participate in the rehabilitation program. Miller ${ }^{[10]}$ feels that 7-12 years is appropriate as children are cooperative for occupational therapy and enough skeletal growth has occurred to prevent recurrence due to increasing muscle tightness secondary to growth. As the child gets older, the contractures become more severe and this may hamper the results of tendon transfer.

Even though surgery is recommended at an early age, older age is not a contraindication as long as the patient tries to use and has voluntary control of the hand..$^{[11]}$ However adults should be evaluated cautiously before surgical planning, because the patients have adapted to the handicap, and surgery must not downgrade function.

\section{Intelligence level}

Assessment of IQ is difficult in a child with cerebral palsy and may not be accurate. ${ }^{[9,12]}$ Many surgeons believe that reconstructive surgery should not be undertaken if the IQ is less than $70 .{ }^{[8,13]}$ Van Heest and colleagues ${ }^{[14]}$ in their study involving 134 patients observed that similar functional improvement (2 level on House scale) can be achieved in the highly motivated patient with fair to good motor control regardless of preoperative mentation, sensibility, and type of cerebral palsy. Leaving the controversy aside most surgeons would agree that the patient must be intelligent enough to interpret what is proposed to him in the way of treatment and to cooperate in postoperative reeducation. IQ is not important when considering patients for procedures which do not require reeducation and training, e.g., wrist fusion.

\section{Summary of the guidelines ${ }^{[4]}$}

- Hygiene should be the primary goal in patients with IQ less than 50, hand placement greater than 5 seconds, and poor sensibility.
- Patients with IQ greater than 50 but poor placement and sensibility should also be considered for procedures to improve the contracted appearance of the limb.

- Patients with IQ greater than 50, hand placement less than 5 seconds, and good sensibility are ideal candidates for functional improvement of the extremity with reconstructive surgery.

\section{Type and extent of neurological involvement}

Only patients with pure spastic type of involvement are amenable for surgical correction. One has to rule out the presence of dystonia or athetosis as the patients with these types of movement disorders do not get better with surgical intervention. The results are unpredictable and some may even get worse after the operation.

\section{Voluntary function}

Voluntary use of the hand is consistently reported as an important predictor of the outcome of operation. ${ }^{[3,14,15]}$ Samilson and Morris ${ }^{[9]}$ wrote that "the patient who has already dissociated his upper limb from bodily functions is not a candidate for reconstruction." The indication for surgery is questionable when patients tend to neglect the affected arm -- "learned disuse." Ideally, surgery to improve function is reserved for those children who have impaired function, but are trying to use the limb. However, if the main indication for surgery is to improve position, it can be done.

\section{Sensation and sensibility}

The usual sensory condition of these patients is the preservation of the basic sensations of touch and pain, and also the ability to recognize the physical characteristics of the touched object- consistency, shape, size, and surface without the aid of sight. The principle sensory deficit is in proprioception and tactile gnosis (two-point discrimination). These sensory deficits

Indian Journal of Plastic Surgery May-August 2011 Vol 44 Issue 2 
make the child not to use his affected upper limb spontaneously unless he is compelled to do so. Thus surgery may improve the function of the hand but does not reintegrate independent function in majority of the cases. The child will still prefer to use the normal hand, using the affected hand only when necessary for bimanual activities. Severe sensory deficit with loss of even the touch and pain sensation is very unusual but makes any attempt at surgical functional restoration ineffective. Although important, sensibility should not in itself be a contraindication to surgery. Many children effectively use hand eye coordination to compensate for defect in steriognosis and proprioception, particularly if they have good voluntary control.

Sensory examination requires, besides the child's cooperation, a certain level of intellectual capacity and language ability. It becomes practical only after $4--5$ years and more complicated tests like 2-PD are reliable only after 6--7years of age. Stereognosis was found to be the most sensitive discriminator of sensibility impairment. ${ }^{[16]}$ Carlson and Brooks ${ }^{[17]}$ found that stereognosis may be affected by hand position and may improve after surgery to improve hand position. Sensation is considered satisfactory when the child identifies at least three out of five objects, can recognize large figures drawn in the palm, and has a two-point discrimination test of no greater than 5--10 mm (according to the child's age). ${ }^{[18]}$

\section{Motivation and environment}

Motivation and parental support are important. Van Heest and colleagues ${ }^{[14]}$ found motivation as the only other significant determinant of outcome other than good motor control.

According to Tonkin, ${ }^{[19]}$ "the ideal candidate is a cooperative 6-years-old child, with stable family support, who has predominantly spastic upper limb deformity, with satisfactory hand sensibility, hemiplegic or monoplegic and without significant neurological deficits."

Finally one must perform a complete general examination (preferably with the help of physician and anesthesiologist) to seek associated neurological disorders and any contraindication to surgery.

\section{Staging of operation}

It is usually appropriate to start proximally as adequate shoulder and elbow function is required to position the hand in space. ${ }^{[15]}$ Zancolli ${ }^{[3]}$ is a proponent of single-stage correction of all the elbow, forearm, wrist, thumb, and finger deformities.

We prefer addressing one deformity in one procedure, instead of doing several different operations at once. This allows us to assess the result of the procedure after rehabilitation and help in planning subsequent procedures. With safer anesthetic techniques, with most operations being done under regional block multiple operations are no more a concern.

\section{Assessment of specific deformities}

In the upper extremity the typical pattern of spastic joint deformity includes shoulder internal rotation, elbow flexion, forearm pronation, wrist flexion and ulnar deviation, thumb-in-palm, and finger swan-neck or clenched fist deformity. Separation into deformities is undoubtedly artificial, but can help to understand and treat the problem more specifically. The following session discusses the assessment and decision-making for correction of pronation deformity at forearm, wrist flexion deformity, swan--neck deformity, and thumb deformities.

\section{Pronation deformity at forearm}

Extreme pronation deformity decreases the sight of the object being grasped and thus obstructs the visual stimulus in these patients with compromised stereognosis.

The ability to supinate the forearm to neutral position improves overall dexterity and function. The hand can then reach the mouth and hair. Simple activities such as clapping the hands and catching balls are possible. ${ }^{20]}$

Palpation of the pronator teres during passive supination of the forearm can identify spasticity. Evaluation for active supination and pronation should also be determined, as well as the position of the forearm at rest.

The classification described by Gschwind and Tonkin ${ }^{[20]}$ provides a sound basis upon which to plan surgery, aimed at improving supination but maintaining pronation, and thus improving function of children with cerebral palsy [Table 2].

We use this classification with following modifications: we divide group 1 patients into two groups depending on the side involved -- right/left. We feel that in a good 
Table 2: Classification of pronation deformity described by Gschwind and Tonkin ${ }^{[20]}$

\begin{tabular}{lll}
\hline Group & Classification & Recommended operative procedure \\
\hline Group 1 & Active supination beyond neutral position & No specific treatment \\
Group 2 & Active supination to less than, or to, neutral position & Pronator quadratus release $+/$ - flexor aponeurotic release \\
Group 3 & $\begin{array}{l}\text { No active supination } \\
\text { Free passive supination }\end{array}$ & Pronator teres transfer \\
Group 4 & $\begin{array}{l}\text { No active supination } \\
\text { No passive supination }\end{array}$ & Pronator quadratus release $+/-$ flexor aponeurotic release \\
\hline
\end{tabular}

functional right hand lack of supination even beyond neutral can be considered a functional problem in Indian culture. Lack of supination beyond neutral makes it difficult to eat with the hand and also unable to take the offerings in the temple or ceremonies (one of the most common complaints of the parents in our patient population). Hence, we would consider them for surgery. In the left hand the same deformity is acceptable. The left hand is used for perineal care, and pronation is important and supination till or past neutral is acceptable both cosmetically and functionally.

In the patients with group 2 type of involvement, we feel that if one is not sure that the deformity is more because of spasticity of the pronators than because of lack of supinator one should do a pronator teres transfer. Because once you have released the pronator quadratus transferring the pronator teres for supination may result in supination deformity. Pronator teres rerouting is a safe option in this group as well. Pronator quadratus retains adequate pronation.

\section{Wrist and hands}

The flexion contracture of the wrist weakens and makes grasping very difficult. This is due to the flexor muscle of the wrist, particularly the flexor carpi ulnaris, remaining contractured during finger flexion (cocontraction). Also the flexion of the wrist slackens the long flexors and hence weakens the grip. This alters the normal pattern of grasping functions of the hand. With better wrist position after surgery the active finger flexion and the grip usually improves.

Zancolli $^{[3]}$ classified the deformities in cerebral palsy as extrinsic and intrinsic based on the predominance of localization of the spasticity of the deformity. Part of his classification which relates to the correction of wrist deformity is widely used [Table 3].

Group 2 patients represent the ideal case for reconstructive surgery since reduction of spasticity and better muscular balance will allow obtaining extension of the fingers with smaller flexion of the wrist and better ability for grasping.

In most spastic hands the possibility of flexing the wrist actively is fundamental for releasing the hand. This implies that wrist fusion is not a very useful operation to improve the function and when the patient has no active wrist flexion and the fingers remain permanently flexed reconstructive surgery is not effective. ${ }^{[3]}$ For the same reason any decision to transfer a wrist flexor to obtain wrist extension must consider the ability to retain wrist flexion. The flexor carpi ulnaris to extensor carpi radialis brevis transfer is well accepted. However, the ability to contract the flexor carpi radialis muscle voluntarily must be assessed. If this is not possible, a wrist extension deformity may result with a consequent inability to extend the fingers.

\section{Swan-neck deformity}

The swan-neck deformity in cerebral palsy can be of two types -- extrinsic and intrinsic. ${ }^{[21]}$ In the extrinsic type the main cause is the overactivity of the long extensor tendons, which is generally due to flexed position of the wrist ${ }^{[21]}$ Overpull of digital extensors causes stretching of the volar plate and eventually hyperextension deformity of the proximal interphalangeal joint without MCP flexion. Once the volar plate is stretched even if the wrist flexion is corrected, the swan-neck deformity persists and can be severe..$^{[22]}$

In the intrinsic type, the main cause is the increased power of traction of the intrinsic muscles and is due to the spasticity of the intrinsic tendons. ${ }^{[21]}$ The patient has substantial MCP joint flexion deformity due to intrinsic spasticity with usually mild swan-neck deformity. ${ }^{[22]}$

It is important to differentiate between these two types of patients because the later requires intrinsic release procedures but the former will require procedures like superficialis tenodesis ${ }^{[23]}$ to prevent hyperextension

Indian Journal of Plastic Surgery May-August 2011 Vol 44 Issue 2 
Table 3: Zancolli's ${ }^{[3]}$ classification of wrist and finger deformity and treatment directions

\begin{tabular}{|c|c|c|c|}
\hline Group & Deformity & Pathology & Treatment guidelines \\
\hline 1 & $\begin{array}{l}\text { Full active finger extension possible with } \\
\text { wrist in almost or completely neutral } \\
\text { extension }\end{array}$ & Spasticity localized to FCU & $\begin{array}{l}\text { Generally no treatment required. } \\
\text { Occasionally tenotomy of the FCU }\end{array}$ \\
\hline 2 & $\begin{array}{l}\text { The fingers can be actively extended } \\
\text { fully but only with wrist flexed. }\end{array}$ & & \\
\hline $2 a$ & $\begin{array}{l}\text { Active extension of the wrist possible } \\
\text { with fingers flexed (Volkmann test) }\end{array}$ & $\begin{array}{l}\text { Spasticity principally localized to the } \\
\text { finger flexors, FCU also may be spastic, } \\
\text { wrist extensors are active }\end{array}$ & $\begin{array}{l}\text { Aponeurotic release of the ventral } \\
\text { muscle of the forearm or fractional } \\
\text { lengthening of the finger flexors, } \\
\text { tenotomy of the FCU if it is spastic }\end{array}$ \\
\hline $2 b$ & $\begin{array}{l}\text { Active extension of the wrist not } \\
\text { possible even with fingers flexed }\end{array}$ & $\begin{array}{l}\text { The wrist extensors are not active, } \\
\text { spasticity of wrist and finger flexors }\end{array}$ & $\begin{array}{l}\text { Transfer of FCU to ECRB with fractional } \\
\text { lengthening of the finger flexors or } \\
\text { aponeurotic release }\end{array}$ \\
\hline 3 & $\begin{array}{l}\text { Active finger extension not possible } \\
\text { even with maximal flexion of the wrist }\end{array}$ & $\begin{array}{l}\text { Marked spasticity of wrist and finger } \\
\text { flexors and paralysis of the wrist } \\
\text { extensors }\end{array}$ & $\begin{array}{l}\text { Poor candidates for surgeries aimed to } \\
\text { improve function } \\
\text { Can consider for operations aimed to } \\
\text { decrease spasticity or improve position }\end{array}$ \\
\hline
\end{tabular}

Table 4: The comprehensive classification of the thumb deformity in cerebral palsy

\begin{tabular}{llll}
\hline Type & Description & Defect & Main component of treatment \\
\hline Type I & Simple metacarpal adduction & AP spastic & Release of the AP \\
Type II & Metacarpal adduction with MCP flexion & AP and FPB spastic & Release of the AP and FPB \\
Type III & $\begin{array}{l}\text { Metacarpal adduction with MCP } \\
\text { hyperextension deformity }\end{array}$ & AP spastic \\
Type IV & $\begin{array}{l}\text { Metacarpal adduction with MCP and IP } \\
\text { flexion }\end{array}$ & AP anstable MCP joint & Release of the AP + Stabilization of the \\
Type V & $\begin{array}{l}\text { IP flexion with less marked metacarpal } \\
\text { adduction } \\
\text { Weakness of extensors with less marked }\end{array}$ & FPL spastic with weak EPL & Weak extensors \\
Type VI & $\begin{array}{l}\text { Weasticity (with or without unstable MCP) } \\
\text { spastic }\end{array}$ & Release of the AP,FPB and FPL \\
\hline
\end{tabular}

of proximal interphalangeal (PIP) joint, lateral band translocation $^{[24]}$ or central slip tenotomy ${ }^{[22]}$ depending on the surgeon's preference and the patient factors.

\section{Thumb}

Thumb involvement is common in cerebral palsy and its management is complex. The thumb held flexed inside the palm impairs grip and grasp and lack of abduction and extension limits the size of the object the patient can grasp. The presence of thumb in the palm also obstructs the function of other fingers. It may even contribute to rejection of the hand and cause problems in hygiene. ${ }^{[25]}$ There are four key things to examine when considering a child with thumb deformity in cerebral palsy for surgical intervention. ${ }^{[4]}$

- Spasticity of adductor and flexor muscles - adductor pollicis (AP), flexor pollicis brevis (FPB), first dorsal interosseous (FDI), flexor pollicis longus (FPL)

- Flaccidity of the extensors and abductors.

- Hypermobility of the metacarpophalangeal (MCP) joint.

- Web space skin contracture.

Indian Journal of Plastic Surgery May-August 2011 Vol 44 Issue 2

\section{Classification of the thumb deformities in cerebral palsy}

The three commonly used classifications for the thumb deformity in cerebral palsy are those described by House and colleagues, ${ }^{[7]}$ Sakellarides et al., ${ }^{[25]}$ and Tonkin. ${ }^{[26]}$

We have observed that in clinical practice there does exist a paralytic type of involvement (weakness of the abductors and extensors without much spasticity of the flexor and adductors) which is not included in the House or Tonkin classification. Also cases of isolated spasticity of the flexor pollicis longus (Tonkin- Extrinsic type) which does not form a part of House classification does exit. To address these issues and to help decision-making we use the following "comprehensive classification" [Table 4].

We have found this classification helpful in assessment and decision-making. It is basically an extended House classification. ${ }^{[7]}$ The first four types are the same as the House classification. Type $\mathrm{V}$ is akin to the Tonkin ${ }^{[26]}$ extrinsic type with FPL spasticity being more marked than the other muscles with weak EPL. These patients 
only require fractional lengthening or z-lengthening of the FPL. Type VI patients have weak abductor and extensors (EPL, EPB, or APL) without any significant spasticity of the flexors. This group represents type1 (weak EPL) and type 3 (APL weakness) of Sakellarides classification. ${ }^{[25]}$ These patients do not require any flexor release and only augmentation of the thumb extension is enough.

There are two important aspects of thumb in palm deformity which need to be addressed - the position of thumb in the palm during fisting and the inability to abduct the thumb when opening the hand. Former usually indicates the spasticity of the adductor and flexors and the later the weakness of the abductor and extensors. The key concept is to rebalance the forces by decreasing the deforming force and augmenting the opposing muscles. Unstable joints may need stabilization and an adequate web should be created. All elements should be addressed at the same sitting, as serial intervention has a high risk of failure or recurrence of deformity. ${ }^{[27]}$

In the patient with voluntary control of extensor pollicis longus and no fixed deformity of the interphalangeal joint rerouting of EPL is an effective procedure. ${ }^{[28]}$ If the extensors are weak they will require augmentation. The transfer of the functioning muscle can be done to abductor pollicis longus (APL), extensor pollicis brevis (EPB) or extensor pollicis longus (EPL). Usually augmentation of EPB is preferred as it brings about the thumb abduction and extension. EPL unless rerouted may still be adducting the thumb and APL does not have any effect over the MCP joint.

Tendon transfer to augment thumb extension leads to further metacarpal adduction and MCPjoint hyperextension if it is very lax. A hyperextension of more than $20^{\circ}$ warrants treatment. ${ }^{[29]}$ Joint stabilization can be done by capsulorrhaphy, sesamoid arthrodesis, or complete fusion of joint. Before fusion a temporary stabilization with K-wire can be useful to assess the consequences of an irreversible procedure. ${ }^{[15]}$

\section{Role of motor blocks ${ }^{[5]}$}

- They differentiate spasticity from fibrous contracture in flexors, adductors, and pronator muscle groups. Injection of spastic pronator teres can help recognize a possible contracture of the interosseous membrane.

- They allow assessment of individual muscles after removing spasticity of the antagonists or cocontractions of adjacent muscles.
- Within the spastic muscle group they help determine which muscles are spastic and which are not.

\section{Role of preoperative therapy}

Splints are a useful adjunct in planning surgical intervention. A patient with a chronically flexed wrist can "test" the functional effect of the wrist in a neutral position by using a wrist splint. Similarly, potential thumb-in-palm correction can be evaluated with a thumb abduction splint, and potential swan-neck deformity correction can be evaluated with an extension block splint. Botulinum toxin may be helpful in predicting the effect of muscle lengthening. Aggressive therapy after injection helps strengthen antagonist muscles.

Counseling the parents and patient has a unique role in the management of a cerebral palsy patient. A surgeon should provide a clear picture of the child's condition and the expected functional status to the parents. The child and parents have to know that the hand will not become normal and whatever interventions we are planning are to improve the function and appearance of the hand. Also they should be told that we are not correcting the basic problem, which is in the brain, but are only trying the address the effects of the problem on the musculoskeletal system to have the best use of the existing functional muscles. It is important to counsel parents and patient regarding this because they usually believe that the hand will be normal after the operation. It is usually harsh for the parents to hear this but the surgeon has to manage this by combining truth with sympathy. The discussion should always end on a positive note since many children can be made better with intervention either in form of therapy or surgery.

\section{REFERENCES}

1. Graham HK, Selber P. Musculoskeletal aspects of cerebral palsy. J Bone Joint Surg Br 2003;85:157-66.

2. Herring JA. Disorders of brain. In: Tachdjian's Pediatric Orthopaedic. $4^{\text {th }}$ ed. Philadelphia: Saunders; 2008. p. 1275-404.

3. Zancolli E. Structural and dynamic bases of hand surgery. $2^{\text {nd }} e d$. Philadelphia: Lippincott; 1979. p. 263-83.

4. Carlson MG. Green's operative hand surgery. In: Green DP, Hotchkiss RN, Pederson WC, Wolfe SW, editors. Cerebral palsy. $5^{\text {th }}$ ed. Philadelphia: Churchill Livingstone; 2005. p. 1197-234.

5. Leclercq C. General assessment of the upper limb. Hand Clin 2003;19:557-64.

6. McConnell K, Johnston L, Kerr C. Upper limb function and deformity in cerebral palsy: A review of classification systems. Dev Med Child Neurol 2011;53:799-805.

7. House JH, Gwathmey FW, Fidler MO. A dynamic approach to the thumb-in-palm deformity in cerebral palsy: Evaluation and results

Indian Journal of Plastic Surgery May-August 2011 Vol 44 Issue 2 
in fifty-six patients. J Bone Joint Surg Am 1981;63:216-25.

8. Green WT, Banks HH. Flexor carpi ulnaris transplant and its use in cerebral palsy. J Bone Joint Surg Am 1962;44:1343-52.

9. Samilson RL, Morris JM. Surgical improvement of the cerebralpalsied upper limb: Electromyographic studies and results of 128 operations. J Bone Joint Surg Am 1964;46:1203-16.

10. Miller F. Cerebral palsy. $1^{\text {st }}$ ed. Wilmington (USA): Springer Publishers; 2005.

11. Manske PR. Cerebral palsy of the upper extremity. Hand Clin 1990;6:697-709.

12. Samilson RL. Principles of assessment of the upper limb in cerebral palsy. Clin Orthop Relat Res 1966;47:105-25.

13. Goldner JL. Upper extremity surgical procedures for patients with cerebral palsy. Instr Course Lect 1979;28:37-66.

14. Van Heest AE, House JH, Cariello C. Upper extremity surgical treatment of cerebral palsy. J Hand Surg Am 1999;24:323-30.

15. Tonkin MA. The growing hand. In: Gupta A, Kay SPJ, Scheker LR, editors. The upper limb in cerebral palsy. London: Mosby; 2000. p. 447-59.

16. Van Heest EA, House J, Putnam M. Sensibility deficiencies in the hands of children with spastic hemiplegia. J Hand Surg Am 1993;18:278-81.

17. Carlson MG, Brooks $C$. The effect of altered hand position and motor skills on stereognosis. J Hand Surg Am 2009;34:896-9.

18. Hoffer MM. The upper extremity in cerebral palsy. AAOS Instructional Course Lecture. St Louis: Mosby; 1979. p. 133-7.

19. Tonkin M. The upper limb in cerebral palsy. Curr Orthop 1995;9:149-55.

20. Gschwind C, Tonkin M. Surgery for cerebral palsy: Part 1: Classification and operative procedures for pronation deformity. J Hand Surg Br 1992;17:391-5.
21. Zancolli E. Structural and dynamic bases of hand surgery. Pathology of the extensor apparatus of the finger: $2^{\text {nd }}$ ed. Philadelphia: Lippincott; 1979. p. 64-104.

22. Carlson MG, Gallagher K, Spirtos M. Surgical treatment of swan neck deformity in hemiplegic cerebral palsy. J Hand Surg Am 2007;32:1418-22.

23. Swanson AB. Surgery of the hand in cerebral palsy and swan neck deformity. J Bone Joint Surg Am 1960;42:951-64.

24. Tonkin MA, Hughes J, Smith KL. Lateral band translocation for swan neck deformity. J Hand Surg Am 1992;17:260-7.

25. Sakellarides HT, Mital MA, Matza RA, Dimakopoulos P. Classification and surgical treatment of the thumb-in-palm deformity in cerebral palsy and spastic paralysis. J Hand Surg Am 1995;20:428-31.

26. Tonkin MA. Surgery for Cerebral palsy part 3: Classification and operative procedures for thumb deformity. J Hand Surg $\mathrm{Br}$ 2001;26:465-70.

27. Goldner JL. Upper extremity tendon transfers in cerebral palsy. Orthop Clin N Am 1974;5:389-414.

28. Manske PR. Redirecting of extensor pollicis longus in the treatment of spastic thumb-in-palm deformity. J Hand Surg Am 1985;10:553-60.

29. Zancolli EA, Zancolli E Jr. Surgical rehabilitation of the spastic upper limb in cerebral palsy. In: Lamb DW, editor. The paralyzed hand. Edinburgh: Churchill Livingstone; 1987. p. 153-68.

How to cite this article: Bhardwaj P, Sabapathy SR. Assessment of the hand in cerebral palsy. Indian J Plast Surg 2011;44:348-56. Source of Support: Nil, Conflict of Interest: None declared. 